\title{
CARACTERIZAÇÃO FÍSICO-MECÂNICA DA MADEIRA DE PEROBA-MICA, Aspidosperma populifolium A. DC. (APOCYNACEAE)
}

\author{
Norman Barros Logsdon*, Zenesio Finger**, Camila Graziela Artioli Borges*** \\ *Eng. Civil, Dr., Faculdade de Engenharia Florestal, UFMT - logsdon@terra.com.br \\ **Eng. Florestal, M.Sc., Faculdade de Engenharia Florestal, UFMT - fingerz@terra.com.br
} ***Acadêmica de Engenharia Florestal, Faculdade de Engenharia Florestal, UFMT - camilagraziela@hotmail.com

Recebido para publicação: 22//05/2006 - Aceito para publicação: 01/10/2007

\begin{abstract}
Resumo
O objetivo deste trabalho foi estudar as características da madeira de peroba-mica. Para descrição dendrológica e identificação da espécie, utilizaram-se os métodos tradicionais da taxonomia. Para caracterização física, utilizou-se o ensaio de estabilidade dimensional proposto por Logsdon (2002). Para caracterização mecânica, utilizou-se a metodologia da atual norma brasileira (NBR 7190/97). A espécie Aspidosperma populifolium A. DC. pertence à família Apocynaceae e distribui-se naturalmente na região amazônica e Centro-Oeste do Brasil. Sua madeira apresentou resistência característica à compressão paralela de $64,98 \mathrm{MPa}$, módulo de elasticidade longitudinal, médio, de $16263 \mathrm{MPa}$, densidade aparente de $0,7259 \mathrm{~g} / \mathrm{cm}^{3}$ e coeficiente de anisotropia dimensional, no inchamento, de 1,9803. Suas características físicas sugerem madeira de qualidade normal, que apresenta alguns defeitos oriundos da secagem, mas pode ser utilizada na fabricação de móveis que aceitem pequenos empenamentos. Suas características mecânicas indicam elevada resistência mecânica (classe de resistência C 60) e aplicação em estruturas de madeira de grande porte.

Palavras-chave: Peroba-mica; Aspidosperma populifolium; resistência; rigidez; densidade.
\end{abstract}

\begin{abstract}
Physical-mechanical characterization of the wood of peroba-mica, Aspidosperma populifolium A. $D C$. (APOCYNACEAE). The objective of this work was to study the characteristics of the wood of peroba-mica. For dendrological description and species identification the traditional taxonomy methods were used. For physical characterization tests, proposed by Logsdon (2002), of dimensional stability were used. For mechanical characterization the methodology described in current Brazilian Code (NBR 7190/97) was used. The species Aspidosperma populifolium A. DC., it belongs to Apocynaceae family and it is distributed naturally at the amazon area and Center-west of Brazil. Its wood presented: characteristic strength to the parallel compression of $64.98 \mathrm{MPa}$; average module of longitudinal elasticity, of $16263 \mathrm{MPa}$; specific gravity of $0.7259 \mathrm{~g} / \mathrm{cm}^{3}$ and coefficient of dimensional anisotropy, in swelling, of 1.9803. Its physical characteristics suggest wood of normal quality, that it presents some defects originating from the dry-out process, but it can be used in the production of pieces of furniture that accept small distortions. Its mechanical characteristics indicate high mechanical strength (resistance class C 60) and application in timber structures of great load. Keywords: Peroba-mica; Aspidosperma populifolium; strength; stiffness; specific gravity.
\end{abstract}

\section{INTRODUÇÃO}

A atual norma brasileira NBR 7190, da ABNT (1997), adota o teor de umidade de referência de $12 \%$. Desse modo, todo resultado de ensaio deve ser reportado a esse teor de umidade. $\mathrm{O}$ antigo método brasileiro MB-26, da ABNT (1940), reeditado como NBR 6230, pela ABNT (1980), utilizava a madeira verde. Assim, os resultados de ensaios do passado não são compatíveis com a atual norma e precisam ser recuperados.

Este trabalho visa suprir essa necessidade e tem por objetivo geral fazer a caracterização físicomecânica da madeira de peroba-mica, Aspidosperma populifolium, além de preparar sua descrição dendrológica. Especificamente, foram obtidas as resistências características à compressão, à tração e ao cisalhamento, todas na direção paralela às fibras. Também foram obtidos os valores médios do módulo de 
elasticidade, da densidade aparente e da densidade básica e foram construídos os diagramas médios de inchamentos e retrações.

As principais características físicas da madeira podem ser obtidas a partir de ensaios de estabilidade dimensional. As dimensões da madeira se alteram substancialmente com a variação da umidade, no intervalo de $0 \%$ até o limite de saturação das fibras. Nesse intervalo, conhecido como intervalo higroscópico, ao aumentar o teor de umidade, as dimensões da madeira aumentam (inchamento), e ao diminuir o teor de umidade, as dimensões diminuem (retração).

Segundo Kollmann; Côté Jr. (1984), o comportamento da madeira ao inchamento volumétrico é o apresentado na figura la e nas expressões (1) e (2). O mesmo comportamento é verificado para as deformações específicas no umedecimento (inchamentos lineares).

$$
\begin{aligned}
& \Delta \mathrm{V}_{\mathrm{i}, \mathrm{U}}=\delta_{\mathrm{Vi}} \cdot \mathrm{U}, \text { para } 0 \% \leq \mathrm{U}<\mathrm{U}_{\mathrm{PI}} \\
& \Delta \mathrm{V}_{\mathrm{i}, \mathrm{U}}=\Delta \mathrm{V}_{\mathrm{i}, \text { sat }}, \text { para } \mathrm{U} \geq \mathrm{U}_{\mathrm{PI}}
\end{aligned}
$$

Onde:

$\mathrm{U}=$ Teor de umidade da madeira, em um instante qualquer do ensaio.

$\Delta \mathrm{V}_{\mathrm{i}, \mathrm{U}}$ e $\Delta \mathrm{V}_{\mathrm{i}, \mathrm{sat}}=$ Variação volumétrica no inchamento, a partir de $\mathrm{U}=0 \%$, até madeira com um teor de umidade qualquer e na situação saturada em água $\left(\mathrm{U} \geq \mathrm{U}_{\mathrm{PI}}\right)$.

$\delta_{\mathrm{Vi}} \quad=$ Coeficiente de inchamento volumétrico, que caracteriza o coeficiente angular da reta inicial do diagrama.

$\mathrm{U}_{\mathrm{PI}} \quad=$ Teor de umidade no ponto de interseção.

A Associação Brasileira de Normas Técnicas (ABNT) admitiu o mesmo comportamento para o estudo da retração (ABNT, 1940; ABNT, 1982). Isso foi um erro, pois o fenômeno não é diretamente associado ao teor de umidade, mas a uma parcela dele, ou seja, ao teor de água de impregnação, que, por sua vez, depende do histórico de umidade.

Logsdon (2000) identificou as diferenças de comportamento entre inchamentos e retrações. Logsdon; Finger (2000) estabeleceram o modelo para o comportamento da madeira na retração volumétrica, apresentado na figura $1 \mathrm{~b}$ e nas expressões (3) e (4), que também se verifica para as deformações específicas na secagem (retrações lineares).

$$
\begin{aligned}
& \text { Para } 0 \% \leq \mathrm{U}<\mathrm{U}_{\text {cond. }}: \Delta \mathrm{V}_{\mathrm{r}, \mathrm{U}}=\Delta \mathrm{V}_{\mathrm{r}, \text { cond. }} \cdot\left(\frac{\mathrm{U}}{\mathrm{U}_{\text {cond. }}}\right)^{\beta_{0, \mathrm{~V}}} \\
& \text { Para } \mathrm{U}_{\text {cond. }} \leq \mathrm{U} \leq \mathrm{U}_{\text {sat. }}: \text { Máximo }\left[\Delta \mathrm{V}_{\mathrm{r}, \mathrm{U}}=\Delta \mathrm{V}_{\mathrm{r}, \text { cond. }} \text { e } \Delta \mathrm{V}_{\mathrm{r}, \mathrm{U}}=\Delta \mathrm{V}_{\mathrm{r}, \text { sat. }} \cdot\left(\frac{\mathrm{U}}{\mathrm{U}_{\text {sat. }}}\right)^{\beta_{1, \mathrm{~V}}}\right]
\end{aligned}
$$

Onde:

$\mathrm{U} \quad=\quad$ Umidade da madeira, em um instante qualquer do ensaio. Em particular, $\mathrm{U}_{\text {sat. }}$ e $\mathrm{U}_{\text {cond. }}$ correspondem, respectivamente, aos teores de umidade do corpo-de-prova saturado em água e condicionado em clima padronizado (temperatura de $20{ }^{\circ} \mathrm{C} \pm 2$ ${ }^{\circ} \mathrm{C}$ e umidade relativa do ar de $65 \% \pm 5 \%$ ).

$\Delta \mathrm{V}_{\mathrm{r}, \mathrm{U}}=$ Variação volumétrica na retração, em um instante qualquer do ensaio. Em particular, $\Delta \mathrm{V}_{\mathrm{r} \text {,sat. }}$ e $\Delta \mathrm{V}_{\mathrm{r} \text {,cond. }}$ correspondem, às variações volumétricas do corpo-de-prova saturado e condicionado, até seco ( $\mathrm{U}=0 \%$ ).

$\beta_{0, \mathrm{~V}}$ e $\beta_{1, \mathrm{~V}}=$ Expoente das curvas. 


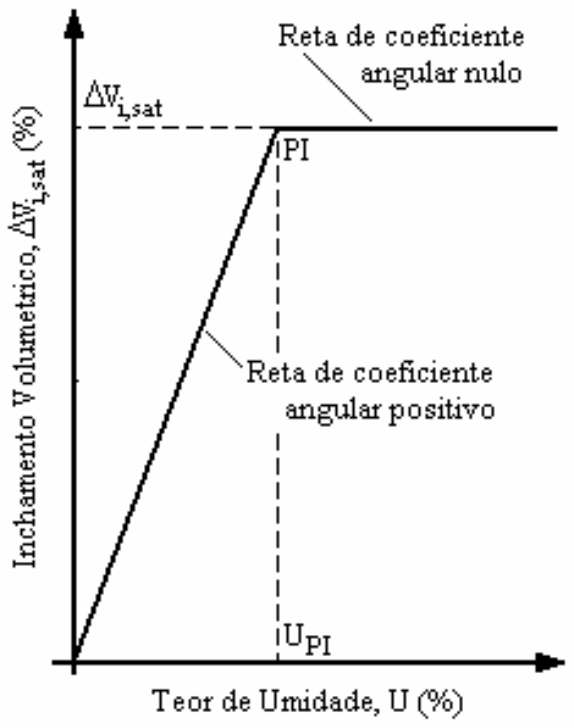

a) Diagrama de inchamento volumétrico

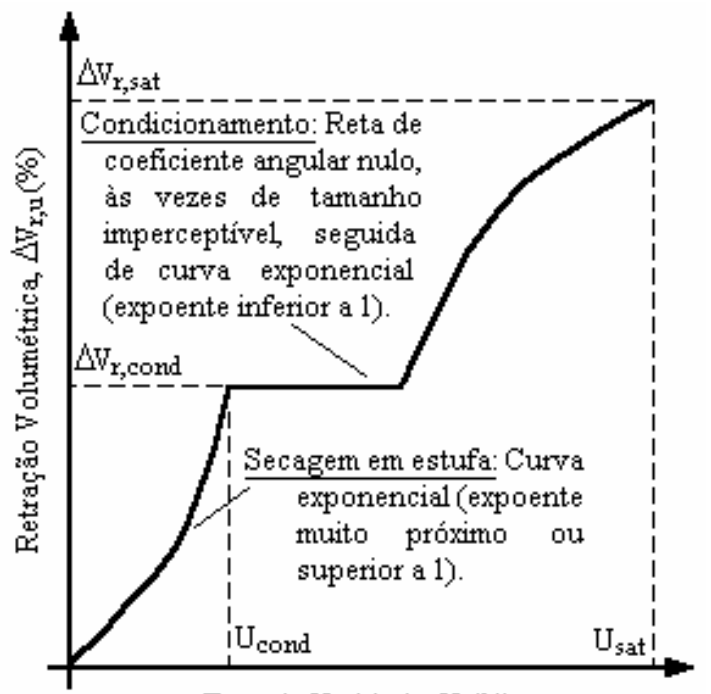

Teor de Utridade, U $(\%)$

b) Diagrama de retração volumétrica

Figura 1. Modelos de comportamento para inchamento e retração volumétrica.

Figure 1. Models of behavior for volumetric swelling and shrinkage.

Kollmann; Côté Jr. (1984) apresentaram um estudo sobre a variação da densidade aparente $\left(\rho_{u}\right)$ com o teor de umidade ( $U$ ) durante o umedecimento da madeira, obtendo o modelo das expressões (5) e (6), função da densidade aparente da madeira seca $\left(\rho_{0}\right)$ e de características do inchamento volumétrico.

$$
\begin{aligned}
& \text { Para } 0 \% \leq U<U_{P I}: \rho_{u}=\rho_{0} \cdot \frac{\left(1+\frac{U}{100}\right)}{\left(1+\frac{\delta_{\mathrm{Vi}} \cdot U}{100}\right)} \\
& \text { Para } U \geq U_{P I}: \rho_{u}=\rho_{0} \cdot \frac{\left(1+\frac{U}{100}\right)}{\left(1+\frac{\Delta V_{i, s a t .}}{100}\right)}
\end{aligned}
$$

Logsdon (2004), de forma análoga a Kollmann; Côté Jr. (1984), estudou a variação da densidade aparente com o teor de umidade durante a secagem da madeira, obtendo o modelo das expressões (7) e (8), função de características da retração volumétrica.

$$
\begin{aligned}
& \text { Para } 0 \% \leq U<U_{\text {cond }}: \rho_{u}=\rho_{0} \cdot\left(1+\frac{U}{100}\right) \cdot\left[1-\frac{\Delta V_{r, \text { cond }}}{100} \cdot\left(\frac{U}{U_{\text {cond }}}\right)^{\beta_{0, \mathrm{~V}}}\right] \\
& \text { Para } U_{\text {cond }} \leq U \leq U_{\text {sat }}: \text { Mínimo }\left[\rho_{u}=\rho_{0} \cdot\left(1+\frac{U}{100}\right) \cdot\left(1-\frac{\Delta V_{r, \text { cond }}}{100}\right) \text { e } \rho_{u}=\rho_{0} \cdot\left(1+\frac{U}{100}\right)\left[1-\frac{\Delta V_{r, s a t}}{100} \cdot\left(\frac{U}{U_{\text {sat }}}\right)^{\beta_{1, V}}\right]\right.
\end{aligned}
$$

O coeficiente de anisotropia, segundo Nock et al. (1975), é usado na indicação da qualidade da madeira quanto aos defeitos oriundos da secagem. Logsdon; Penna (2004) ampliaram os estudos de Nock et al. (1975) e apresentaram a tabela 1. 
A atual NBR 7190, da ABNT (1997), adota a umidade de referência de 12\%. Assim, todos os resultados de ensaio devem ser reportados a essa umidade. A NBR 7190/97 apresenta formulário para a correção da resistência e rigidez da madeira, mas não para sua densidade aparente. Logsdon (1998), estudando o assunto, concluiu que o formulário apresentado na NBR 7190/97 não é válido para todas as classes de resistência ou todos os tipos de solicitação e apresentou formulário alternativo, que também permite a correção da densidade aparente. Esse formulário é apresentado nas expressões (9) e (10) e na tabela 2.

$$
\begin{aligned}
& \mathrm{f}_{12}=\mathrm{f}_{\mathrm{U} \%} \cdot\left[1+\frac{\alpha \cdot(\mathrm{U} \%-12)}{100}\right] ; \mathrm{E}_{12}=\mathrm{E}_{\mathrm{U} \%} \cdot\left[1+\frac{\alpha \cdot(\mathrm{U} \%-12)}{100}\right] \\
& \rho_{12}=\rho_{\mathrm{U} \%}+\rho_{\mathrm{U} \%} \cdot\left[\left(1-\delta_{\mathrm{V}}\right) \cdot \frac{(12-\mathrm{U} \%)}{100}\right], \operatorname{com} \delta_{\mathrm{V}}=\frac{\Delta \mathrm{V}}{\mathrm{U} \%} \text { e } \Delta \mathrm{V}=\frac{\mathrm{V}_{\mathrm{U} \%}-\mathrm{V}_{\text {sec a }}}{\mathrm{V}_{\text {sec a }}} \cdot 100 \%
\end{aligned}
$$

Onde:

$$
\begin{array}{ll}
\mathrm{f}_{12} \text { e } \mathrm{f}_{\mathrm{U} \%}= & \begin{array}{l}
\text { Resistência, em determinada solicitação, respectivamente, aos teores de umidade } \\
\text { de } 12 \% \text { e } \mathrm{U} \% .
\end{array} \\
\mathrm{E}_{12} \text { e } \mathrm{E}_{\mathrm{U}} & \begin{array}{l}
\text { Módulo de elasticidade longitudinal, respectivamente, aos teores de umidade de } \\
=
\end{array} \\
\alpha & \begin{array}{l}
12 \% \text { e } \mathrm{U} \% . \\
=
\end{array} \quad \text { Deeficiente de correção, fornecido na tabela } 2 . \\
= & \text { Coeficiente de retratibilidade volumétrica. } \\
\rho_{12} \text { e } \rho_{\mathrm{U} \%} & \text { Retração volumétrica, para a variação de umidade entre } \mathrm{U} \% \text { e } 0 \% . \\
\delta_{\mathrm{V}} & \text { Volume do corpo-de-prova, respectivamente, ao teor de umidade } \mathrm{U} \% \text { e da } \\
\Delta \mathrm{V} & \text { madeira seca } \mathrm{U}=0 \% .
\end{array}
$$

\begin{tabular}{|c|c|c|c|}
\hline \multicolumn{2}{|c|}{ Coeficiente de anisotropia em: } & \multirow{2}{*}{$\begin{array}{l}\text { Qualidade da } \\
\text { madeira }\end{array}$} & \multirow{2}{*}{ Utilização indicada para a madeira } \\
\hline Retração, $A_{r}$ & Inchamento, $\mathrm{A}_{\mathrm{i}}$ & & \\
\hline Até 1,50 & Até 1,54 & Excelente & $\begin{array}{c}\text { Móveis finos, esquadrias, barcos, aparelhos musicais, } \\
\text { aparelhos de esporte etc. }\end{array}$ \\
\hline 1,50 a 2,00 & 1,54 a 2,10 & Normal & $\begin{array}{l}\text { Estantes, mesas, armários, enfim, usos que permitam } \\
\text { pequenos empenamentos. }\end{array}$ \\
\hline Acima de 2,00 & Acima de 2,10 & Ruim & $\begin{array}{l}\text { Construção civil (observadas as características } \\
\text { mecânicas), carvão, lenha etc. }\end{array}$ \\
\hline
\end{tabular}

Tabela 1. Coeficiente de anisotropia dimensional, qualidade e uso da madeira.

Table 1. Coefficient of dimensional anisotropy, quality and use of the wood.

Segundo a ABNT (1997), para a caracterização das propriedades de resistência, podem ser utilizados os três seguintes procedimentos: caracterização completa da resistência da madeira serrada, caracterização mínima da resistência da madeira serrada e caracterização simplificada da resistência da madeira serrada. Para a caracterização das propriedades de rigidez, podem ser utilizados os dois seguintes procedimentos: caracterização completa da rigidez da madeira e caracterização simplificada da rigidez da madeira.

Tabela 2. Valores do coeficiente de correção, $\alpha$.

Table 2. Values of the correction coefficient, $\alpha$.

\begin{tabular}{lc}
\hline Propriedade de resistência ou rigidez & Coeficiente de correção, $\alpha$ \\
\hline Resistência à compressão paralela às fibras, $\mathrm{f}_{\mathrm{c} 0}$ & 3,5 \\
Resistência à tração paralela às fibras, $\mathrm{f}_{\mathrm{t} 0}$ & 2,0 \\
Resistência ao cisalhamento paralelo às fibras, $\mathrm{f}_{\mathrm{v} 0}$ & 2,5 \\
Módulo de elasticidade longitudinal, $\mathrm{E}_{\mathrm{c} 0}$ & 2,5 \\
\hline
\end{tabular}




\section{MATERIAL E MÉTODOS}

O material foi coletado de três árvores da espécie Aspidosperma populifolium, no município de Cotriguaçu (MT). De cada árvore, foi coletado material botânico para a identificação e descrição da espécie e uma amostra do fuste, na região do DAP (diâmetro à altura do peito), de aproximadamente 90 $\mathrm{cm}$ de comprimento, da qual foram retiradas, a partir de uma prancha diametral, quatro barras de seção 6 $\mathrm{cm}$ x $6 \mathrm{~cm}$. De cada barra foi retirado um corpo-de-prova para cada ensaio previsto. Na figura 2, são apresentados o esquema de retirada e as dimensões dos corpos-de-prova. Para cada tipo de ensaio, foram retirados 12 corpos-de-prova, correspondendo à amostragem mínima prevista na NBR 7190, da ABNT (1997).

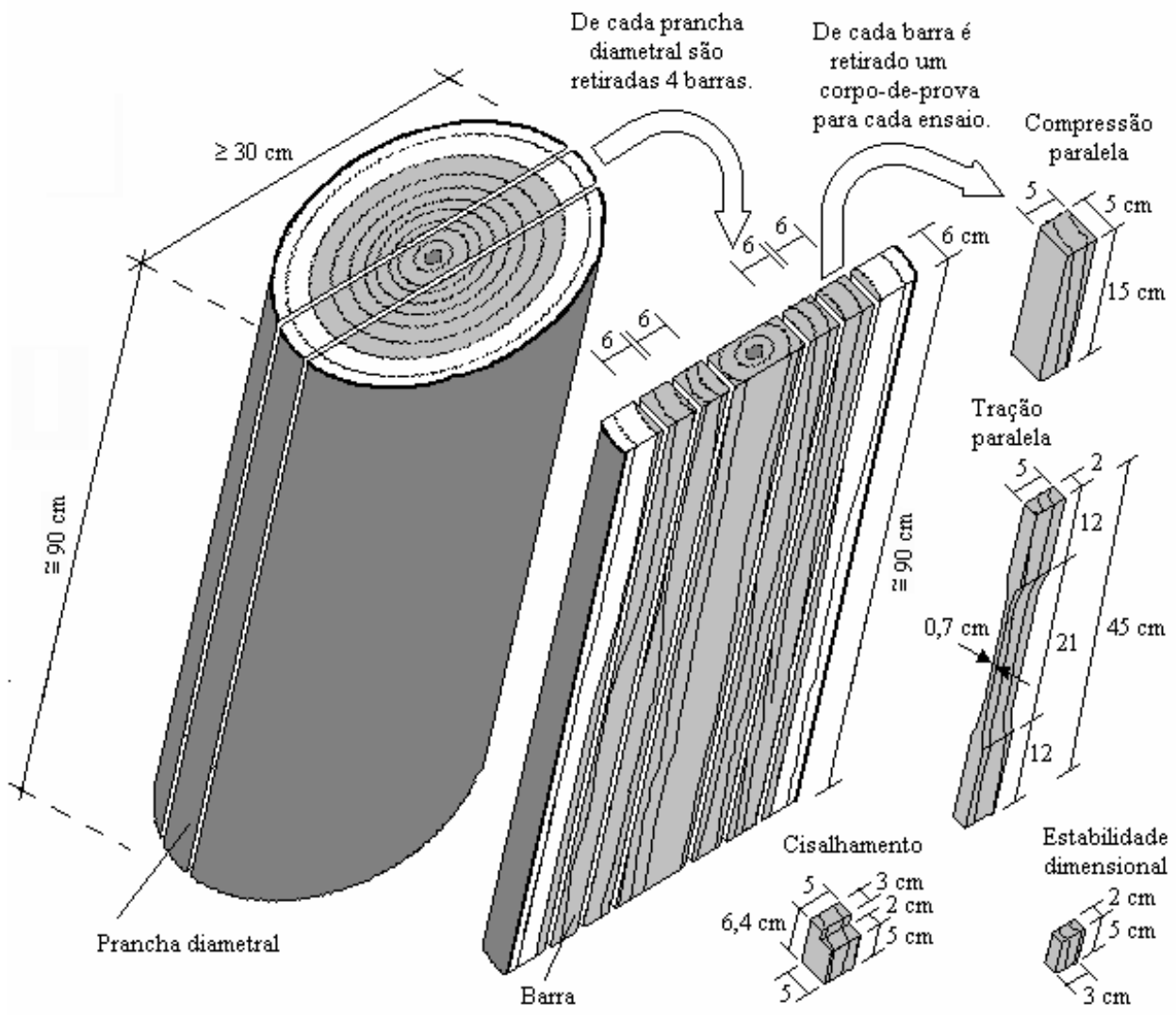

Figura 2. Esquema de retirada e dimensões dos corpos-de-prova.

Figure 2. Scheme to saw and dimensions of the specimens.

Para a descrição dendrológica da espécie, foram coletadas informações sobre as particularidades do caule, da copa, das folhas, da casca e da madeira.

A identificação da espécie foi realizada através dos padrões clássicos utilizados pela taxonomia, com base em caracteres morfológicos florais e vegetativos, pela comparação de exsicatas coletadas com material catalogado no Herbário Central da Universidade Federal de Mato Grosso e pela consulta a outras instituições e especialistas. A nomenclatura da espécie foi citada de acordo com o Index Kewensis.

A caracterização física foi baseada na metodologia proposta por Logsdon (2002) para revisão da atual NBR 7190/97, que inclui os ensaios de inchamento e retração. De cada árvore foi separado um corpo-de-prova como amostra de controle.

O ensaio de estabilidade dimensional tem quatro fases: secagem prévia (corpos-de-prova são secados no ar), encharcamento (corpos-de-prova são submersos em água destilada), condicionamento (corpos-de-prova são secados no ar) e secagem em estufa (corpos-de-prova são secados em estufa). As 
três primeiras fases foram realizadas em sala de climatização, com temperatura de $20 \pm 2{ }^{\circ} \mathrm{C}$ e umidade relativa do ar de $65 \pm 5 \%$, e a última em uma estufa de secagem e esterilização, com temperatura de $103 \pm$ $2{ }^{\circ} \mathrm{C}$.

As dimensões e as massas dos corpos-de-prova da amostra de controle foram avaliadas ao longo de cada fase (a cada 20 minutos no primeiro dia e diariamente nos demais). $\mathrm{O}$ término da fase era detectado pela observação da constância da massa dos corpos-de-prova (variação inferior a $0,5 \%$ em um intervalo mínimo de seis horas).

As avaliações de massa e de dimensões para os demais corpos-de-prova foram feitas nos seguintes instantes do ensaio: 1 ) após a secagem prévia $\left(U=U_{\text {início }} \cong 12 \%\right)$, que corresponde ao início do ensaio de inchamento; 2) no fim da fase de encharcamento $\left(U=U_{\text {sat. }}\right.$ ), que corresponde ao fim do ensaio de inchamento e ao início do ensaio de retração, quando a madeira estava saturada em água; 3 ) após um dia na sala de climatização $\left(U=U_{1 d-S C}\right)$, que é um ponto intermediário da fase de condicionamento; 4) no fim da fase de condicionamento ( $\mathrm{U}=\mathrm{U}_{\text {cond }} \cong 12 \%$ ), que corresponde ao início da fase de secagem em estufa, quando a madeira estava condicionada; 5) após uma hora secando em estufa $\left(U=U_{1 h-e s t}\right)$, que é um ponto intermediário da fase de secagem em estufa; e 6) no fim da secagem em estufa ( $U=0 \%$ ), quando a madeira estava seca.

A fase de encharcamento e o instante do ensaio correspondente ao corpo-de-prova completamente seco compõem o ensaio de inchamento.

As fases de condicionamento e de secagem em estufa compõem o ensaio de retração.

Caso algum corpo-de-prova apresentasse defeito durante a secagem, seria descartado.

Para a caracterização mecânica, inicialmente foi feita uma secagem prévia das barras das quais foram retirados os corpos-de-prova, aos moldes da descrita para o ensaio de estabilidade dimensional, com o intuito de fazer os ensaios mecânicos em corpos-de-prova com teor de umidade entre $0 \%$ e $20 \%$. Os métodos utilizados foram os descritos na NBR 7190 (ABNT, 1997).

Os ensaios de cisalhamento e tração paralela foram realizados em máquina universal de ensaios, a uma velocidade de carregamento de $2,5 \mathrm{MPa} / \mathrm{min}$ e $10 \mathrm{MPa} / \mathrm{min}$, respectivamente. Desses ensaios, foram anotadas a força de ruptura, a área do corpo-de-prova que resistia ao carregamento e a massa do corpo-de-prova, no fim do ensaio e após completa secagem. Assim, foi possível obter as correspondentes tensões de ruptura e o teor de umidade dos corpos-de-prova.

Os ensaios de compressão paralela também foram realizados em máquina universal de ensaios, com uma velocidade de carregamento de $10 \mathrm{MPa} / \mathrm{min}$. Para medir as deformações, foram fixados, aos corpos-de-prova, dois extensômetros eletrônicos, um de cada lado, com base de leitura de $10 \mathrm{~cm}$, curso de $2 \mathrm{~mm}$ e sensibilidade de $0,5 \mu \mathrm{m}$ (micrômetro). Os ensaios foram realizados com dois ciclos iniciais de carga e um final. Os ciclos iniciais se limitaram a $50 \%$ da estimativa da tensão de ruptura (obtida em ensaio de outro corpo-de-prova). No ciclo final (terceiro), cujo carregamento foi aumentado gradativamente até a ruptura, foram obtidos os resultados. No início e fim de cada ciclo, a carga foi mantida constante durante 30 segundos, como recomenda a NBR 7190 (ABNT, 1997). Desses ensaios, no início/fim de cada ciclo e a cada $10 \%$ da tensão de ruptura estimada, no ciclo final, foram anotadas a carga e a deformação. Também foram anotadas a força de ruptura, a área da seção transversal do corpode-prova e a massa do corpo-de-prova, no fim do ensaio e após completa secagem. Assim, foi possível obter a tensão de ruptura, o módulo de elasticidade longitudinal e o teor de umidade dos corpos-de-prova no instante do ensaio.

Para reportar os resultados ao teor de umidade de referência, foram utilizados os resultados de Logsdon (1998).

Os valores característicos de tensões foram obtidos com o operador definido na NBR 7190 (ABNT, 1997), ou seja, o valor obtido da equação (11).

$$
\operatorname{Máximo}\left[\mathrm{x}_{1} ; 0,7 . \mathrm{x}_{\mathrm{wm}} \text { e } \mathrm{x}_{\mathrm{wk}}=\left(2 . \frac{\mathrm{x}_{1}+\mathrm{x}_{2}+\ldots+\mathrm{x}_{\frac{\mathrm{n}}{2}-1}}{\frac{\mathrm{n}}{2}-1}-\mathrm{x}_{\frac{\mathrm{n}}{2}}\right)\right]
$$


Onde:

$\mathrm{x}_{\mathrm{i}}=$ Resistência, obtida no ensaio do corpo-de-prova i. Os valores $\mathrm{x}_{\mathrm{i}}$ são ordenados de forma crescente, de modo que $\mathrm{x}_{1}$ é a menor resistência obtida nos ensaios.

$\mathrm{x}_{\mathrm{wm}}=$ Valor médio das resistências obtidas nos ensaios.

$\mathrm{x}_{\mathrm{wk}} \quad=$ Operador para $\mathrm{o}$ valor característico da resistência.

$\mathrm{n} \quad=$ Número de corpos-de-prova ensaiados (tamanho da amostra).

\section{RESULTADOS E DISCUSSÃO}

A espécie Aspidosperma populifolium pertence à família Apocynaceae e distribui-se naturalmente na região amazônica e no Centro-Oeste do Brasil, nos estados do Amazonas, Pará, Rondônia e Mato Grosso. Ocorre principalmente nas Florestas Ombrófilas Densas e Abertas, Submontanas e dificilmente ultrapassa $30 \mathrm{~m}$ de altura, podendo alcançar até $75 \mathrm{~cm}$ de diâmetro. Apresenta fuste alto e muito cilíndrico, com a copa geralmente concentrada no ápice da árvore. A ramificação é cimosa e a copa é aberta e capitata umbeliforme. Suas folhas são simples, alternas, com exsudação leitosa pegajosa na abcissão foliar. Sua casca é profundamente fissurada e corticosa, com ritidoma pardo a acastanhado, de deiscência granular. A casca viva, após oxidação, adquire a cor amarelo queimado e apresenta gosto muito amargo. A madeira dessa espécie é densa $\left(\rho_{a p, 12 \%} \cong 0,73 \mathrm{~g} / \mathrm{cm}^{3}\right)$, com cerne marrom-acastanhado claro, com veios mais escuros, sendo pouco distinto do alburno, que geralmente é mais claro. Escurece em exposição o ar, é lisa ao tato e recebe acabamento opaco a pouco lustroso. Sua textura é fina, a grã é ondulada, o cheiro é indistinto e o gosto é amargo. É resistente ao ataque de fungos e xilófagos. Floresce de agosto a setembro e frutifica de novembro a dezembro. Sua madeira é muito utilizada na construção civil, como vigas, caibros e ripas para pisos, e na indústria de carpete de madeira, como lâmina de capa, na fabricação de móveis de qualidade superior, em acabamento de interiores e para fabricação de portas e batentes.

As principais características físicas da madeira de peroba-mica (Aspidosperma populifolium) são apresentadas nas tabelas 3 a 6 . As características mecânicas são apresentadas na tabela 7 .

Tabela 3. Características físicas da madeira de peroba-mica (Aspidosperma populifolium), nos ensaios de inchamentos.

Table 3. Physical characteristics of the wood of peroba-mica (Aspidosperma populifolium), in the swelling test.

\begin{tabular}{|c|c|c|c|c|c|c|c|c|}
\hline & \multirow{2}{*}{$\begin{array}{c}\text { Umidade } \\
\text { de sa- } \\
\text { turação }\end{array}$} & \multicolumn{3}{|c|}{$\begin{array}{l}\text { Coeficientes de inchamentos, nas } \\
\text { direções: }\end{array}$} & \multicolumn{3}{|c|}{$\begin{array}{l}\text { Inchamentos totais, } \\
\text { nas direções: }\end{array}$} & \multirow{2}{*}{$\begin{array}{l}\text { C. de } \\
\text { Aniso- } \\
\text { tropia }\end{array}$} \\
\hline & & Radial & Tangencial & Volumétrica & Radial & Tangencial & Volumétrica & \\
\hline & $\mathrm{U}_{\text {sat. }}(\%)$ & $\delta_{\mathrm{i}, 2}$ & $\delta_{\mathrm{i}, 3}$ & $\delta \mathbf{v}_{\mathbf{i}}$ & $\varepsilon_{\mathrm{i}, 2}(\%)$ & $\varepsilon_{\mathrm{i}, 3}(\%)$ & $\Delta \mathbf{V}_{\mathrm{i}}(\mathbf{\%})$ & $\mathbf{A}_{\mathbf{i}}$ \\
\hline Número & 12 & 12 & 12 & 12 & 12 & 12 & 12 & 12 \\
\hline Média & 66,85 & 0,1403 & 0,2567 & 0,3953 & 3,7735 & 7,3656 & 11,5722 & 1,9803 \\
\hline D. Padrão & 4,5812 & 0,0190 & 0,0426 & 0,0399 & 0,5699 & 0,6423 & 1,1227 & 0,2506 \\
\hline \multicolumn{9}{|c|}{ Intervalo de Confiança da Média } \\
\hline $\mathrm{t}(95 \%)$ & 2,2010 & 2,2010 & 2,2010 & 2,2010 & 2,2010 & 2,2010 & 2,2010 & 2,2010 \\
\hline Lim. Inf. & 63,94 & 0,1283 & 0,2296 & 0,3700 & 3,4114 & 6,9575 & 10,8589 & 1,8211 \\
\hline Lim. Sup. & 69,76 & 0,1524 & 0,2838 & 0,4207 & 4,1356 & 7,7737 & 12,2856 & 2,1395 \\
\hline
\end{tabular}

Os modelos especificados foram verificados com os dados obtidos com a amostra de controle e todos apresentaram regressão com bom coeficiente de determinação $\left(0,7440 \leq \mathrm{R}^{2} \leq 0,9856\right)$. Assim, foram construídas as curvas, com os resultados médios de três árvores, apresentadas na figura 3.

Os ensaios de caracterização mecânica forneceram os valores característicos de algumas resistências e o valor médio da rigidez da madeira de peroba-mica (Aspidosperma populifolium). Para o cálculo de estruturas de madeiras, são necessários outros valores, que podem ser obtidos com auxílio de expressões definidas na NBR 7190 (ABNT, 1997). Essas expressões e correspondentes resultados são apresentados nas expressões (12) a (15). 
Tabela 4. Características físicas de peroba-mica (Aspidosperma populifolium), na fase de condicionamento dos ensaios de retrações.

Table 4. Physical characteristics of the wood of peroba-mica (Aspidosperma populifolium), in the conditioning phase of the shrinkage test.

\begin{tabular}{|c|c|c|c|c|c|c|c|}
\hline & \multicolumn{3}{|c|}{$\begin{array}{l}\text { Retrações totais, madeira saturada, nas } \\
\text { direções: }\end{array}$} & \multicolumn{3}{|c|}{$\begin{array}{l}\text { Expoentes das curvas, no trecho final } \\
\text { (diagramas de retrações): }\end{array}$} & \multirow{2}{*}{$\begin{array}{l}\text { C. de } \\
\text { Aniso- } \\
\text { tropia }\end{array}$} \\
\hline & Radial & Tangencial & Volumétrica & Radial & Tangencial & Volumétrica & \\
\hline & $\varepsilon_{\mathrm{r}, 2}(\%)$ & $\varepsilon_{\mathrm{r}, 3}(\%)$ & $\Delta \mathbf{V}_{\mathrm{r}}(\%)$ & $\beta_{1 \mathrm{r}, 2}(\%)$ & $\beta_{1 \mathrm{r}, 3}(\%)$ & $\beta_{1, \mathrm{Vr}}(\%)$ & $\mathbf{A}_{\mathbf{r}}$ \\
\hline Número & 12 & 12 & 12 & 12 & 12 & 12 & 12 \\
\hline Média & 3,6336 & 6,8573 & 10,3636 & 0,0935 & 0,0953 & 0,0968 & 1,9131 \\
\hline D. Padrão & 0,5293 & 0,5580 & 0,9025 & 0,0604 & 0,0564 & 0,0484 & 0,2341 \\
\hline \multicolumn{8}{|c|}{ Intervalo de Confiança da Média } \\
\hline$t(95 \%)$ & 2,2010 & 2,2010 & 2,2010 & 2,2010 & 2,2010 & 2,2010 & 2,2010 \\
\hline Lim. Inf. & 3,2973 & 6,5027 & 9,7902 & 0,0551 & 0,0594 & 0,0661 & 1,7644 \\
\hline Lim. Sup. & 3,9699 & 7,2118 & 10,9371 & 0,1319 & 0,1311 & 0,1275 & 2,0619 \\
\hline
\end{tabular}

Tabela 5. Características físicas de peroba-mica (Aspidosperma populifolium), na fase de secagem dos ensaios de retrações.

Table 5. Physical characteristics of the wood of peroba-mica (Aspidosperma populifolium), in the oven dry-out phase of the shrinkage test.

\begin{tabular}{|c|c|c|c|c|c|c|c|}
\hline & \multirow{2}{*}{$\begin{array}{c}\text { U.de } \\
\text { condicio- } \\
\text { namento }\end{array}$} & \multicolumn{3}{|c|}{$\begin{array}{l}\text { Retrações em condicionamento, } \\
\text { nas direções: }\end{array}$} & \multicolumn{3}{|c|}{$\begin{array}{l}\text { Expoentes das curvas no trecho inicial } \\
\text { (diagramas de retrações): }\end{array}$} \\
\hline & & Radial & Tangencial & Volumétrica & Radial & Tangencial & Volumétrica \\
\hline & $\mathrm{U}_{\text {cond. }}(\%)$ & $\varepsilon_{\mathrm{r}, 2 \text { cond. }}(\%)$ & $\varepsilon_{\mathrm{r}, 3 \text { cond. }}(\%)$ & $\Delta \mathbf{V}_{\mathrm{r}, \text { cond. }}(\%)$ & $\beta_{0 \mathrm{r}, 2}(\%)$ & $\beta_{0 \mathrm{r}, 3}(\%)$ & $\beta_{0, \mathrm{Vr}}(\%)$ \\
\hline Número & 12 & 12 & 12 & 12 & 12 & 12 & 12 \\
\hline Média & 14,04 & 2,4736 & 4,5040 & 7,1239 & 1,2635 & 1,7319 & 1,5138 \\
\hline D. Padrão & 0,2091 & 0,2915 & 0,3019 & 0,6575 & 0,1553 & 0,4986 & 0,3249 \\
\hline \multicolumn{8}{|c|}{ Intervalo de Confiança da Média } \\
\hline $\mathrm{t}(95 \%)$ & 2,2010 & 2,2010 & 2,2010 & 2,2010 & 2,2010 & 2,2010 & 2,2010 \\
\hline Lim. Inf. & 13,89 & 1,2053 & 3,0959 & 4,8189 & 0,4721 & 0,7561 & 0,6003 \\
\hline Lim. Sup. & 14,46 & 1,6264 & 3,8857 & 5,7251 & 0,6721 & 1,0422 & 0,8038 \\
\hline
\end{tabular}

Tabela 6. Outras características físicas da madeira de peroba-mica (Aspidosperma populifolium).

Table 6. Others physical characteristics of the wood of peroba-mica (Aspidosperma populifolium).

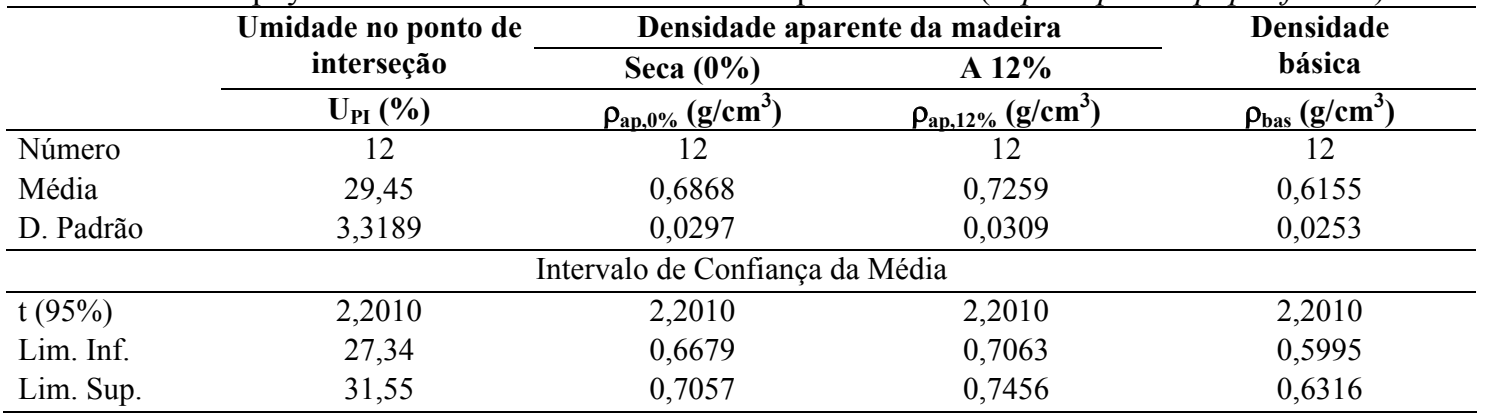

$$
\begin{array}{ll}
\mathrm{f}_{\mathrm{c} 90, \mathrm{k}} / \mathrm{f}_{\mathrm{c} 0, \mathrm{k}}=0,25 \Rightarrow \mathrm{f}_{\mathrm{c} 90, \mathrm{k}}=0,25 \cdot \mathrm{f}_{\mathrm{c} 0, \mathrm{k}}, \text { portanto: } & \mathrm{f}_{\mathrm{c} 90, \mathrm{k}}=0,25 \cdot 64,98 \Rightarrow \mathrm{f}_{\mathrm{c} 90, \mathrm{k}}=16,95 \mathrm{MPa} \\
\mathrm{f}_{\mathrm{e} 0, \mathrm{k}} / \mathrm{f}_{\mathrm{c} 0, \mathrm{k}}=1,00 \Rightarrow \mathrm{f}_{\mathrm{e} 0, \mathrm{k}}=1,00 \cdot \mathrm{f}_{\mathrm{c} 0, \mathrm{k}}, \text { portanto: } & \mathrm{f}_{\mathrm{e} 0, \mathrm{k}}=1,00 \cdot 64,98 \Rightarrow \mathrm{f}_{\mathrm{e} 0, \mathrm{k}}=64,98 \mathrm{MPa} \\
\mathrm{f}_{\mathrm{e} 90, \mathrm{k}} / \mathrm{f}_{\mathrm{c} 0, \mathrm{k}}=0,25 \Rightarrow \mathrm{f}_{\mathrm{e} 90, \mathrm{k}}=0,25 \cdot \mathrm{f}_{\mathrm{c} 0, \mathrm{k}}, \text { portanto: }: & \mathrm{f}_{\mathrm{e} 90, \mathrm{k}}=0,25 \cdot 64,98 \Rightarrow \mathrm{f}_{\mathrm{e} 90, \mathrm{k}}=16,25 \mathrm{MPa} \\
\mathrm{E}_{\mathrm{c} 90, \mathrm{~m}}=\frac{1}{20} \cdot \mathrm{E}_{\mathrm{c} 0, \mathrm{~m}}, \text { portanto: } & \mathrm{E}_{\mathrm{c} 90, \mathrm{~m}}=\frac{16263}{20} \Rightarrow \mathrm{E}_{\mathrm{c} 90, \mathrm{~m}}=813,15 \mathrm{MPa}
\end{array}
$$


Onde:
$\mathrm{f}_{\mathrm{c} 0, \mathrm{k}}$ e $\mathrm{f}_{\mathrm{e} 0, \mathrm{k}}$
$=$ Resistências características à compressão e ao embutimento paralelos às fibras.
$\mathrm{f}_{\mathrm{c} 90, \mathrm{k}}$ e $\mathrm{f}_{\mathrm{e} 90, \mathrm{k}}$
$=$ Resistências características à compressão e ao embutimento normais às fibras.
$\mathrm{E}_{\mathrm{c} 0, \mathrm{~m}}$ e $\mathrm{E}_{\mathrm{c} 90, \mathrm{~m}}$
$=$ Valores médios dos módulos de elasticidade longitudinal e transversal.

Tabela 7. Características mecânicas da madeira de peroba-mica (Aspidosperma populifolium).

Table 7. Mechanical characteristics of the wood of peroba-mica (Aspidosperma populifolium).

\begin{tabular}{|c|c|c|c|c|}
\hline & $\begin{array}{c}\text { Módulo de } \\
\text { elasticidade } \\
\text { longitudinal }\end{array}$ & $\begin{array}{c}\text { Resistência à } \\
\text { compressão } \\
\text { paralela às fibras }\end{array}$ & $\begin{array}{l}\text { Resistência à tração } \\
\text { paralela às fibras }\end{array}$ & $\begin{array}{l}\text { Resistência ao } \\
\text { cisalhamento } \\
\text { paralelo às fibras }\end{array}$ \\
\hline & $\mathrm{E}_{\mathrm{c} 0,12 \%}(\mathrm{MPa})$ & $\mathrm{f}_{\mathrm{c} 0,12 \%}(\mathrm{MPa})$ & $\mathbf{f}_{\mathrm{t} 0,12 \%}(\mathrm{MPa})$ & $\mathbf{f}_{\mathrm{v} 0,12 \%}(\mathrm{MPa})$ \\
\hline Número & 12 & 12 & 12 & 12 \\
\hline Média & 16263 & 64,83 & 87,63 & 11,21 \\
\hline D. Padrão & 2064,37 & 3,1770 & 34,1964 & 1,4846 \\
\hline Coef. de Variação & 0,13 & 0,05 & 0,39 & 0,13 \\
\hline Valor característico & --- & 64,98 & 61,34 & 10,35 \\
\hline \multicolumn{5}{|c|}{ Intervalo de Confiança da Média } \\
\hline $\mathrm{t}(95 \%)$ & 2,2010 & 2,2010 & 2,2010 & 2,2010 \\
\hline Lim. Inferior & 14951 & 62,81 & 65,90 & 10,27 \\
\hline Lim. Superior & 17574 & 66,85 & 109,36 & 12,15 \\
\hline
\end{tabular}

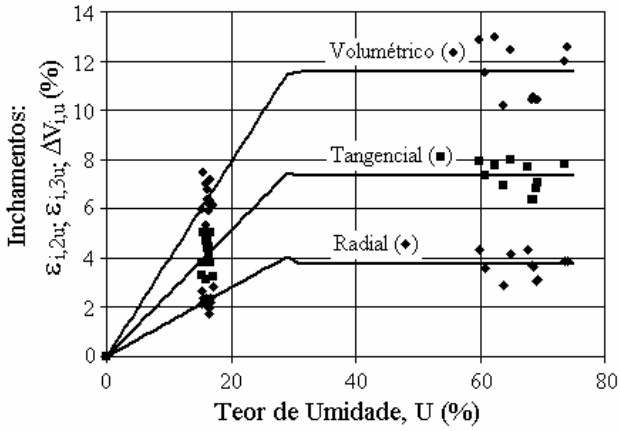

a) Diagrama de inchamentos

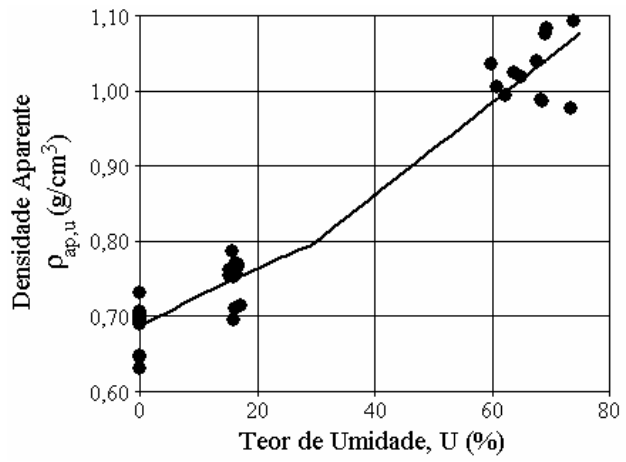

c) Densidade aparente $X$ teor de umidade durante o umedecimento da madeira

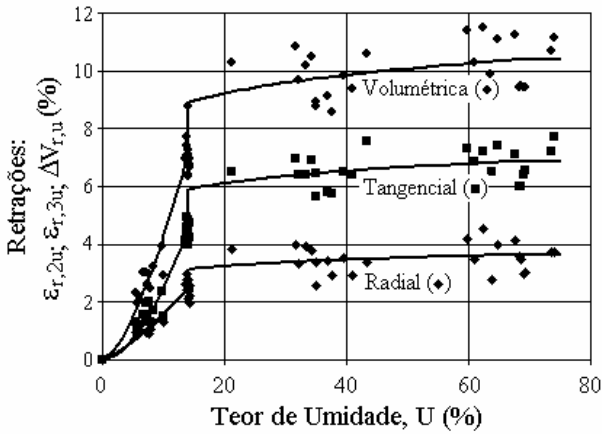

b) Diagrama de retrações

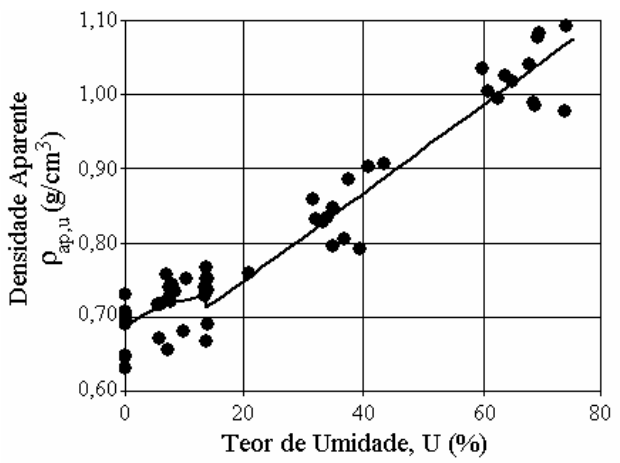

d) Densidade aparente $\mathrm{X}$ teor de umidade durante a secagem da madeira

Figura 3. Curvas obtidas para a madeira de peroba-mica (Aspidosperma populifolium), valores médios de três árvores.

Figure 3. Curves obtained for the wood of peroba-mica (Aspidosperma populifolium), and average values of three. 
Não foram encontrados na literatura estudos utilizando metodologia atual de ensaio para madeira de peroba-mica (Aspidosperma populifolium).

\section{CONCLUSÕES}

Os resultados indicam que a madeira de peroba-mica (Aspidosperma populifolium) possui elevada resistência mecânica e pode ser utilizada em estruturas de madeira de grande porte, pois o valor característico da resistência à compressão paralela às fibras $\left(\mathrm{f}_{\mathrm{c} 0, \mathrm{k}}=64,98 \mathrm{MPa}\right)$ a posiciona na classe de resistência C 60, maior classe de resistência definida pela NBR 7190, (ABNT, 1997) para as dicotiledôneas.

Para cálculo de estruturas de madeira executadas com madeira de peroba-mica (Aspidosperma populifolium), podem ser utilizados os valores característicos e médios, apresentados nas tabelas 3 a 7 e expressões (12) a (15).

Os coeficientes de anisotropia dimensional no inchamento $\left(\mathrm{A}_{\mathrm{i}}=1,9803\right)$ e na retração $(\mathrm{Ar}=1,9131)$ indicam que a madeira de peroba-mica (Aspidosperma populifolium) é considerada normal e pode ser utilizada na fabricação de móveis que aceitem pequenos empenamentos, como mesas, cadeiras, estantes, etc. Recomendam-se, para trabalhos futuros, estudos sobre acabamentos, adesão e trabalhabilidade da madeira dessa espécie, permitindo uma indicação mais segura para a fabricação de móveis.

As tabelas 3 a 7 apresentadas anteriormente fornecem dados que podem ser utilizados como indicativos de qualidade por outros setores da indústria da madeira.

Resultados obtidos para as relações $\mathrm{f}_{\mathrm{c} 0, \mathrm{k}} / \mathrm{f}_{\mathrm{t} 0, \mathrm{k}}$ e $\mathrm{f}_{\mathrm{v} 0, \mathrm{k}} / \mathrm{f}_{\mathrm{c} 0, \mathrm{k}}$, respectivamente, de 1,06 e 0,16 , diferem dos valores adotados pela atual NBR 7190 (ABNT, 1997), de 0,77 e 0,12. Isso indica a necessidade de revisão das expressões definidas na atual norma brasileira e recomenda a continuidade de trabalhos como este, a fim de subsidiar a tomada de decisão dos autores da norma a esse respeito.

\section{AGRADECIMENTOS}

Os autores agradecem à Fundação de Amparo à Pesquisa do Estado de Mato Grosso (FAPEMAT), pelo financiamento e pela bolsa da estudante envolvida no trabalho, e ao Sr. Artemio Richter, proprietário da Madeireira Richter Ltda., pelo fornecimento da madeira e apoio na fase de coleta de material.

\section{REFERÊNCIAS}

ASSOCIAÇÃO BRASILEIRA DE NORMAS TÉCNICAS. MB 26: ensaios físicos e mecânicos de madeiras. Rio de Janeiro, 1940.

ASSOCIAÇÃO BRASILEIRA DE NORMAS TÉCNICAS. NBR 6230: ensaios físicos e mecânicos de madeiras. Rio de Janeiro, 1980.

ASSOCIAÇÃO BRASILEIRA DE NORMAS TÉCNICAS. NBR 7190: projeto de estruturas de madeiras. São Paulo, 1997.

KOLlmanN, F. F. P.; CÔTÉ JR., W. A. Principles of wood science and technology: Solid Wood. Berlin: Springer-Verlag, 1968-1984. v.1.

LOGSDON, N. B.; FINGER, Z. Modelos para especificação das curvas dos diagramas de retrações e inchamentos. Madeira: Arquitetura e Engenharia. São Carlos, ano 1, n. 3, 1CD-ROM, Set/Dez. 2000.

LOGSDON, N. B. Influência da umidade nas propriedades de resistência e rigidez da madeira. 174 p. Tese (Doutorado em Engenharia de Estruturas) - Escola de Engenharia de São Carlos, Universidade de São Paulo, São Carlos, 1998. 
LOGSDON, N. B. Sobre os ensaios de retrações e inchamentos. Madeira: Arquitetura e Engenharia, São Carlos, SP, ano 1, n. 2, p. 19-24, maio/ago. 2000.

LOGSDON, N. B. Estabilidade dimensional: Propostas para revisão da NBR 7190/97. In: ENCONTRO BRASILEIRO EM MADEIRAS E EM ESTRUTURAS DE MADEIRA, 8., Uberlândia, MG. Anais... Uberlândia: FECIV-UFU, 2002. 1 (CD-ROM).

LOGSDON, N. B. Variação da densidade aparente da madeira com sua umidade, modelagem teórico experimental. Madeira: Arquitetura e Engenharia, São Carlos, SP, ano 4, n. 12, 1CD-ROM, jan/abr. 2004.

LOGSDON, N. B.; PENNA, J. E. Análise comparativa entre os coeficientes de anisotropia dimensional da madeira, no inchamento e na retração. Agricultura Tropical, Cuiabá, v. 8, n. 1, 2004.

NOCK, H. P.; RICHTER, H. G.; BURGER, L. M. Tecnologia da madeira. Curitiba: UFPR, 1975. 21p. 